\title{
Collapse of triangular channels in a soft elastomer
}

\author{
Daniel Tepáyotl-Ramírez, ${ }^{1}$ Tong Lu, ${ }^{1}$ Yong-Lae Park, ${ }^{2}$ and Carmel Majidi ${ }^{1}$ \\ ${ }^{1}$ Department of Mechanical Engineering, Carnegie Mellon University, Pittsburgh, Pennsylvania 15213, USA \\ ${ }^{2}$ Wyss Institute for Biologically-Inspired Engineering, Harvard University, Cambridge, \\ Massachusetts 02138, USA
}

(Received 25 October 2012; accepted 15 January 2013; published online 28 January 2013)

\begin{abstract}
We extend classical solutions in contact mechanics to examine the collapse of channels in a soft elastomer. These channels have triangular cross-section and collapse when pressure is applied to the surrounding elastomer. Treating the walls of the channel as indenters that penetrate the channel base, we derive an algebraic mapping between pressure and cross-sectional area. These theoretical predictions are in strong agreement with results that we obtain through finite element analysis and experimental measurements. This is accomplished without data fitting and suggests that the theoretical approach may be generalized to a broad range of cross-sectional geometries in soft microfluidics. @ 2013 American Institute of Physics. [http://dx.doi.org/10.1063/1.4789762]
\end{abstract}

Soft-matter electronics and microfluidic devices contain no rigid materials and are instead composed of channels of fluid embedded in a soft elastic medium. In contrast to conventional electronics, these elastomer-based technologies are intrinsically soft, stretchable, and elastically compatible with natural human tissue and organs. Applications range from soft-matter electronics and sensor arrays for wearable computing ${ }^{1-4}$ to soft lab-on-a-chip devices for cell sorting and DNA analysis. ${ }^{7,8}$ Channels are typically filled with conductive liquid (e.g., liquid phase metal alloy), compressed gas, or biological fluids and embedded in a silicone elastomer, e.g., polydimethylsiloxane (PDMS).

Because the surrounding elastomer is soft, these microfluidic channels deform easily and can collapse under external pressure. In the case of valves, ${ }^{9}$ pressure sensors, ${ }^{10-12}$ and particle filtration, ${ }^{8}$ channel collapse under external pressure introduces unique soft-matter functionality. Triangular crosssectional geometries, such as those presented in Fig. 1, are especially important because of the nonlinear dependency that allows for relatively dramatic change in cross-sectional area under moderate external pressure. This nonlinear dependency allows for relatively dramatic changes in cross-sectional area under modest loads and can be used to selectively filter and trap nanoparticles and manipulate single DNA molecules. ${ }^{8}$ Also, Park et al. show that liquid alloy-filled microchannels with triangular geometries exhibit relatively high linearity and low hysteresis for resistive soft-matter pressure sensing. ${ }^{12}$

Here, we examine the collapse of two classes of triangular cross-sectional geometries: equilateral (Fig. 1(a)) and concave (Fig. 1(b)) triangles. When pressure is applied, the surrounding elastomer deforms and the channel walls collapse, as shown in Fig. 1 for the equilateral (Fig. 1(c)) and concave (Fig. 1(d)) geometries. We perform experimental measurements and finite element analysis (FEA) for channels embedded in an incompressible (Poisson's ratio $\nu=0.5$ ) silicone elastomer (Ecoflex 00-30; Smooth-On, Inc.) that has an elastic modulus $E=125 \mathrm{kPa}^{10}$ These results are in strong agreement with closed-form theoretical predictions that we derive using a unique approach based on analogies to elastic deformation in contact mechanics. This theory does not depend on any fitting parameters and can be generalized to predict deformation in a broad range of symmetric crosssectional geometries.

As illustrated in Fig. 2, we treat the channel side walls as elastic indenters that penetrate the channel base with a force proportional to the total vertical pressure exerted above the wall. Since the walls are at an angle to the vertical direction, this force will be approximately equal to the vertical component of the internal stress surrounding the channel multiplied by the channel width. It is important to note that the side walls are analogous to only one half of an indenter. In order to utilize classical results from contact mechanics for the penetration of an elastic indenter into an elastic halfspace, we must assume that there is a neighboring channel on the opposite side of the indenter. That is, instead of modeling a single channel, this theoretical analogy is most accurate for modeling an array of channels.

In the case of a triangular cross-section of width $w$ (Fig. 1(a)), the side walls resemble one half of an equilateral triangular wedge. Therefore, the collapse of the channel can be examined by studying the analogous case of the penetration of an elastic wedge into an elastic halfspace, as

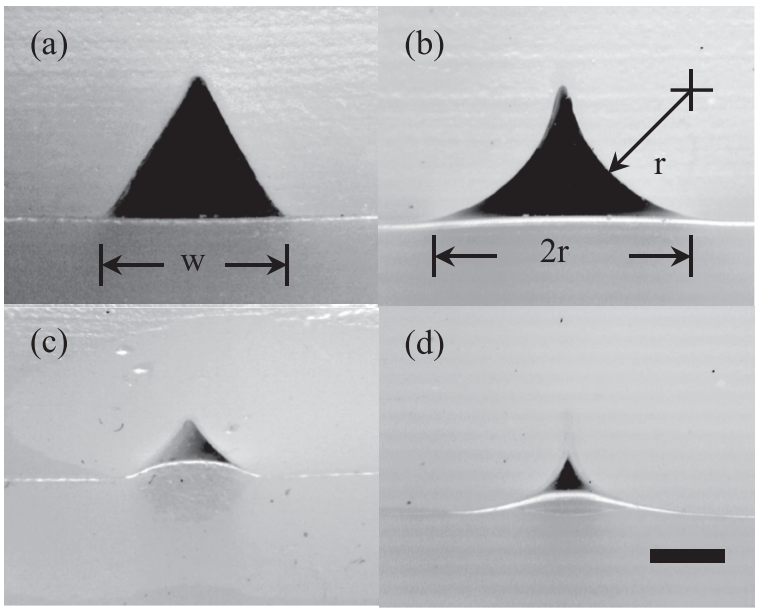

FIG. 1. Channel cross-sections with (a) equilateral and (b) concave triangle geometries. The collapsed (c) equilateral and (d) concave channels when pressure is vertically applied on the surrounding elastomer. The scale bar corresponds to $1 \mathrm{~mm}$. 
(a)

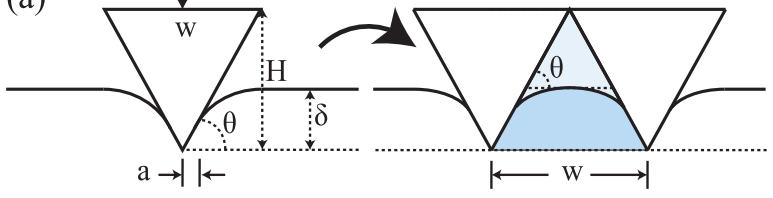

(b)

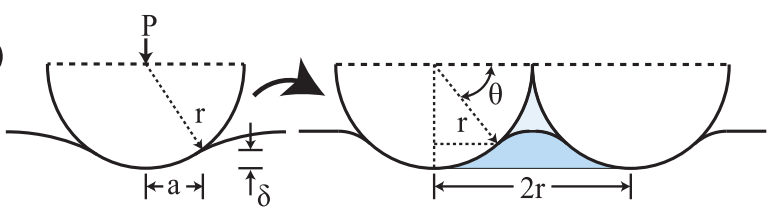

FIG. 2. Representation of a (a) wedge and (cylindrical) indenter used to model the penetration of the channel side walls into the channel based when pressure is applied to the surrounding elastomer.

illustrated in Fig. 2(a). Applying a pressure $p$ to the surface of the elastomer leads to internal stress with a vertical component $\sigma_{z z}=: \sigma$. Assuming that the channel is sufficiently close to the area where stress is applied, $\sigma \approx p$. If the depth $z$ or lateral position $x$ of the channel with respect to the center of applied pressure are large compared to the width $a$ of the contact area, then $\sigma$ may be approximated as $\chi p$, where $\chi=\chi(x, z)$ is obtained using Boussinesq's method for $2 \mathrm{D}$ plane strain elasticity. ${ }^{10}$ However, $\chi \approx 1$ when $x=0$ and $z$ is sufficiently small (i.e., $z<a$ ) and so, without loss of generality, we let $\sigma=p$.

For the wedge indenter in Fig. 2(a), this pressure is concentrated into a force per unit length $P$, which is given by the expression $P=\sigma w$. This pressure causes the wedge to indent the underlying elastic halfspace with a penetration depth $\delta$ and contact width $2 a$. For a wedge and substrate with an elastic modulus $E$ and Poisson's ratio $\nu, a=2\left(1-\nu^{2}\right) P / E \tan \theta$, where $\theta=\pi / 3$ is the angle of the side wall. ${ }^{13}$ The channel has a height $H=w \tan \theta / 2$, and so $a$ may be expressed as $\left(1-\nu^{2}\right) \sigma w^{2} / E H$. Referring to geometry in Fig. 2(a), the cross-sectional area of the deformed channel is approximated as

$$
A \approx \frac{2 H}{w}\left(\frac{w}{2}-a\right)^{2} .
$$

Finally, for an initial area $A_{0}=w H / 2$ and substituting the expressions for $P$ and $a$, the relative change in cross-sectional area may be expressed as

$$
\frac{\Delta A}{A_{0}}=1-\left(1-\frac{2\left(1-\nu^{2}\right) \sigma w}{E H}\right)^{2} .
$$

We use a similar approach to model the collapse of a channel with a concave triangular geometry (Fig. 1(b)). In this case, the side walls correspond to a cylindrical indenter of radius $r$ that is loaded with a force per unit length $P=2 \sigma r$ (Fig. 2(b)). This leads to a penetration depth $\delta=8\left(1-\nu^{2}\right)$ $P / \pi E,{ }^{14}$ where again $E$ and $\nu$ are the elastic modulus and Poisson's ratio, respectively, and a contact width $2 a$, where $a=\sqrt{r^{2}-(r-\delta)^{2}}$. Next, referring to Figure 2(b), the crosssectional area of the channel is approximated as

$$
A \approx 2\left\{(r-a)(r-\delta)+\frac{1}{2} a(r-\delta)-\frac{1}{2} \theta r^{2}\right\}
$$

where $\theta=\arcsin (1-\delta / r)$. Finally, for an initial area of $A_{0}=2 r^{2}-\pi r^{2} / 2$, the relative change in area is calculated as

$$
\frac{\Delta A}{A_{0}}=1-\frac{2\left\{(r-a)(r-\delta)+(r-\delta) a / 2-r^{2} \theta / 2\right\}}{r^{2}(2-\pi / 2)} .
$$

By substituting the expressions for $\delta$ and $a$ into Eq. (4), we obtain a closed-form theoretical prediction for $\Delta A / A_{0}$.

We validated the predictions in Eqs. (2) and (4) with a series of FEA simulations and experiments. As demonstrated in Figs. 3 and 4, the results obtained from FEA and experiments are in strong agreement with the theory. This agreement is accomplished without the aid of data fitting and suggests that the mathematical assumptions used to derive the closed-form expressions for $\Delta A / A_{0}$ may potentially be extended to other symmetric channel geometries where solutions for analogous problems in contact mechanics exist.

We used Abaqus FEA 6.11 (Dassault Systèmes) to perform a two-dimensional plane-strain FEA simulation for the elastic deformation of an incompressible elastomer ( $E=125 \mathrm{kPa}$ and $\nu=0.49$ ) embedded with either an equilateral or concave triangular channel. In order to be consistent with the experimental measurements, we selected elastomer dimensions of $16 \mathrm{~mm}$ by $75 \mathrm{~mm}$ and channel dimensions of $w=4 \mathrm{~mm}$ and $r=4 \mathrm{~mm}$ for the equilateral and concave triangle, respectively (Fig. 5). We also varied the surface pressure $p$ from 0 to $70 \mathrm{kPa}$ over a region of length $a=20 \mathrm{~mm}$ centered 5.69 and $6 \mathrm{~mm}$ above the top of the equilateral and concave triangle openings, respectively. The left and right edges of the FEA model are constrained in the horizontal direction. The bottom edge is constrained in the vertical direction. Frictionless contact options are applied to the channel edges. After convergence is obtained through successive mesh refinement, we saved the image of the deformed opening for each increment of applied pressure. Image processing was performed in MATLAB R2011a (MathWorks, Inc.) and gave the relative change in cross-sectional area for both the equilateral and concave triangle geometries. These estimates of $\Delta A / A_{0}$ can be directly compared with the theoretical predictions in Eqs. (2) and (4) calculated for $E=125 \mathrm{kPa}$ and $\nu=0.5$.

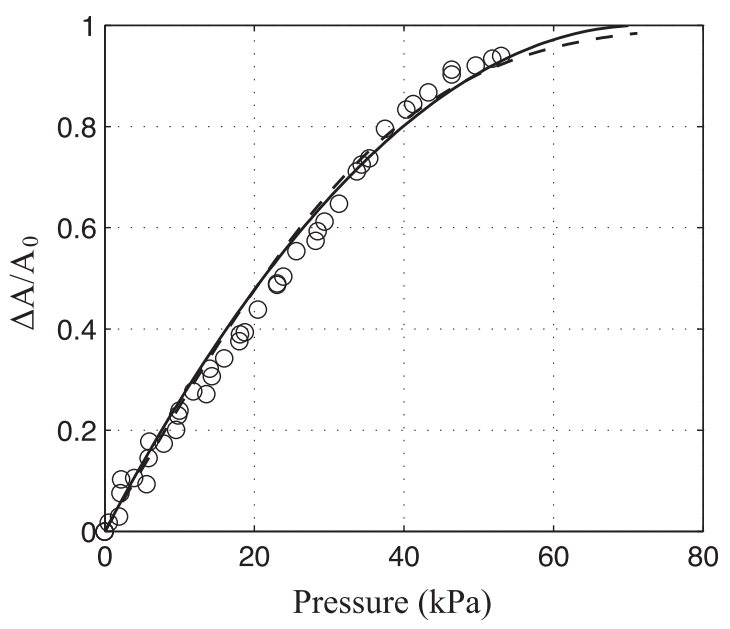

FIG. 3. Theory (-), FEA (--), and experimental data $\left(^{\circ}\right)$ for a equilateral triangle cross section; $E=125 \mathrm{kPa}, \nu=0.5$, and $w=4 \mathrm{~mm}$. 


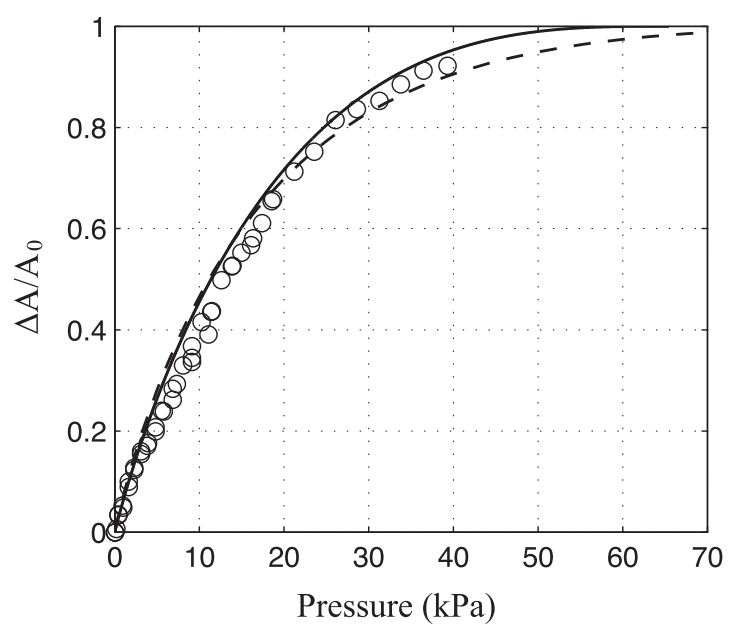

FIG. 4. Theory (-), FEA (--), and experimental data $\left({ }^{\circ}\right)$ for a concave triangle cross section; $E=125 \mathrm{kPa}, \nu=0.5$, and $r=4 \mathrm{~mm}$.

In addition to FEA modeling, we verified our theoretical predictions with experimental measurements performed on molded silicone substrates. The samples have a length of $75 \mathrm{~mm}$, a width of $75 \mathrm{~mm}$, and thickness of $16 \mathrm{~mm}$. The width of the equilateral triangular channel $w$ and the radius of the concave triangle $r$ are both $4 \mathrm{~mm}$. The samples were fabricated by pouring uncured Ecoflex 00-30 (Smooth-On, Inc.) into molds produced with a desktop 3D printer (Objet24, Objet Ltd.). Each sample was placed against the wall of a rigid, acrylic glass case, and cross-sectional deformation was monitored with a digital microscope (Firefly GT800-Oasis Scientific, Inc.). By placing the samples against the walls, all the deformation occurs in the plane of the cross-section. Frictional stresses between the wall and elastomer are assumed to be small relative to the applied stresses, so they are not incorporated in this analysis. We controlled the vertical pressure by placing the acrylic case and sample on an electronic scale (5000 g-OHAUS Corporation) and pressing down on the sample with a $20 \times 20 \mathrm{~mm}$ square block attached to a height gauge. To vary the pressure, we adjusted the gauge height. The corresponding images, examples of which are shown in Fig. 1, were then analyzed with IMAGEJ (National Institute of Health) to determine the relative change in cross-sectional area.

The theoretical prediction (solid curve), FEA data (dashed curve), and experimental values (open circles) are

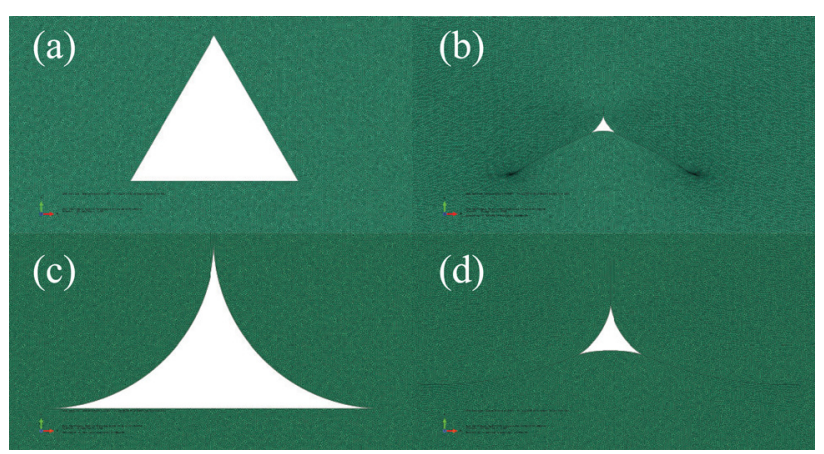

FIG. 5. FEA images depicting the (a) undeformed and (b) deformed equilateral triangle cross section, and the (c) undeformed and (d) deformed concave triangle cross section. The scale bar corresponds to $2 \mathrm{~mm}$. plotted in Figures 3 and 4 for the two cross-sectional geometries. In both cases, we observe strong agreement between the theory, FEA simulation, and experimental results. This is accomplished without the aid of data fitting and is based entirely on the prescribed channel dimensions ( $w=r=4 \mathrm{~mm}$ ), elastic modulus $E=125 \mathrm{kPa}$, Poisson's ratio $\nu=0.5$, and applied surface pressure $p$.

Experimental data are only collected for pressures up to $55 \mathrm{kPa}$ and $40 \mathrm{kPa}$ for the equilateral and concave triangles, respectively. Beyond these values, the channel image resolution was too low to perform accurate image processing. Below these values, we measure less than $5 \%$ error between the experimental measurements, FEA results, and theoretical predictions. Also, multiple loading and unloading cycles demonstrate repeatability and low hysteresis. Although there is high accuracy between the theoretical, experimental, and FEA data, the range of pressure is limited to only $70 \mathrm{kPa}$. This corresponds to the pressure required to completely collapse the channel and can be adjusted by selecting a more elastically rigid material.

From Eqs. (2) and (4), it is apparent that we can increase the resistance of the channels to collapse by increasing the elastic modulus of the surrounding elastomer. For example, replacing Ecoflex 00-30 with PDMS rubber could dramatically increase the pressure required to collapse the channel. It has been reported that PDMS can be engineered to have an elastic modulus as high as $4 \mathrm{MPa}{ }^{15}$ To examine the influence of the elastic modulus, we repeated our FEA study for $E=2 \mathrm{MPa}, \nu=0.49$, and the same geometries as before. In these analyses, the pressure required to collapse the channels increases and the theoretical predictions matches the new curve. We calculate $2 \%$ and $5 \%$ maximum error in $\Delta A / A_{0}$ between the theoretical and FEA predictions for the equilateral and concave triangles, respectively. Conversely, if a material with lower elastic modulus (e.g., Ecoflex 5) is used, then a lower pressure is required to change the crosssectional area. This approach may be useful for sensors, valves, electrical switches, and other microfluidics applications where functionality depends on channel collapse.

Although internal pressure is ignored, channels will typically be filled with a fluid that exerts traction on the channel walls. This pressure may be significant for pneumatic actuation in soft inflatable robots ${ }^{5,6}$ or other applications in which the fluid has large hydrostatic pressure or is tightly confined. In most applications, however, this internal pressure will be negligible compared to the large elastic stresses induced by external surface pressure. For example, in the case of softmatter electronics ${ }^{1-3,10-12}$ and microfluidics, ${ }^{8,9}$ external pressure is only applied over a relatively small portion of the microfluidic channel. Therefore, the fluid will be free to flow out of the compressed region and maintain its relatively low nominal hydrostatic pressure. Nonetheless, when hydrostatic pressure is large or the fluid is tightly confined, the theory must be modified to include the influence of internal pressure.

Future work should also address the potentially significant role of adhesion, especially in the case of micro-scale channels with smooth channel walls. In general, adhesion may increase $\Delta A / A_{0}$ and lead to contact hysteresis. In this study, however, the elastomer is cast in a $3 \mathrm{D}$ printed mold that has microscale roughness. This surface roughness 
prevents the millimeter-scale channel walls from adhering, which is why we do not observe significant hysteresis and can ignore adhesion in the theory. Also, the theory and FEA are based on linear elastic deformation and predict internal strains that never exceed 35\%. Even during complete channel collapse, the nominal strains in the neighborhood of the channel are well within the linear elastic regime of a soft silicone elastomer. Therefore, we are confident in the assumption of linear elasticity for the materials and pressure ranges of interest. Nonetheless, for more rigid elastomers or higher loads, it may be necessary to incorporate non-linear elasticity into the model. Finally, the indentation law used to examine deformation of the concave triangular channel (Fig. 1(b)) requires $r \gg a{ }^{14}$ However, this condition is violated for large pressures such that $a$ is on the order of $r$ and the channel is nearly collapsed. This likely contributes to the significant deviation between FEA and theory observed in Fig. 4 for pressures $p>30 \mathrm{kPa}$.

In closing, we introduce a method for predicting the collapse of a channel with triangular cross-sections when pressure is applied to the surrounding elastic medium. A closed-form algebraic relationship between pressure $(p)$ and the relative change in cross-sectional area $\left(\Delta A / A_{0}\right)$ is obtained by treating the channel walls as indenters that penetrate the channel base as pressure is applied. By recasting an otherwise complex two-dimensional plane strain elasticity problem into a tractable contact mechanics problem, we derive theoretical predictions that are consistent with results obtained with FEA simulation and experimental measurement. In general, we expect the theoretical predictions to be most accurate at low pressures since the derivations are based on linear elastic deformation and classical contact mechanics. Nonetheless, this simplified approach provides a reasonable first-order approximation that eliminates the need for numerical computation. Moreover, this approach may be generalized to other cross-sections with wall geometries that resemble the surface of an indenter. We anticipate that the theories presented here could be further developed to include internal hydrostatic pressure from embedded fluid or reaction forces from trapped particles. Future efforts could also focus on adhesion between collapsing channel walls and how this might allow a channel to remain closed even after pressure is removed.

${ }^{1}$ R. K. Kramer, C. Majidi, and R. J. Wood, Proc. IEEE ICRA (2011), 11031107.

${ }^{2}$ R. D. Wong, J. D. Posner, and V. J. Santos, Sensors Actuators, A 179, $62-$ 69 (2012).

${ }^{3}$ S. Cheng and Z. Wu, Adv. Func. Mater. 21, 2282-2290 (2011).

${ }^{4} \mathrm{~K}$. Noda, E. Iwase, K. Matsumoto, and I. Shimoyama, Proc. IEEE ICRA (2010), 4212-4217.

${ }^{5}$ R. F. Shepherd, F. Ilievski, W. Choi, S. A. Morin, A. A. Stokes, A. D. Mazzeo, X. Chen, M. Wang, and G. M. Whitesides, Proc. Natl. Acad. Sci. USA 108, 20400-20403 (2011).

${ }^{6} \mathrm{~K}$. Suzumori, S. Endo, T. Kanda, N. Kato, and H. Suzuki, IEEE ICRA (2007), 4975-4980.

${ }^{7}$ S. R. Quake and A. Scherer, Science 290, 1536-1540 (2000).

${ }^{8}$ D. Huh, K. L. Mills, X. Zhu, M. A. Burns, T. D. Thouless, and S. Takayama, Nature Mater. 6, 424-428 (2007).

${ }^{9}$ M. A. Unger, H.-P. Chou, T. Thorsen, A. Scherer, and S. R. Quake, Science 288, 113-116 (2000).

${ }^{10}$ Y.-L. Park, C. Majidi, R. Kramer, P. Bérard, and R. J. Wood, J. Micromech. Microeng. 20, 125029 (2010).

${ }^{11}$ Y.-L. Park, B.-R. Chen, and R. J. Wood, IEEE Sens. J. 12, 2711-2718 (2012).

${ }^{12}$ Y.-L. Park, D. Tepayotl-Ramirez, R. J. Wood, and C. Majidi, Appl. Phys. Lett. 101, 191904 (2012).

${ }^{13}$ I. N. Sneddon, Fourier Transforms, 1st ed. (McGraw-Hill, New York, 1951), pp. 436-438.

${ }^{14}$ K. L. Johnson, Contact Mechanics (Cambridge University Press, Cambridge, 1985), pp. 99-101.

${ }^{15}$ D. Fuard, T. Tzvetkova-Chevolleau, S. Decossas, P. Tracqui, and P. Schiavone, Microelectronic Eng. 85, 1289-1293 (2008). 